\title{
A Comparison of Functional Networks Derived From Representational Similarity, Functional Connectivity, and Univariate Analyses
}

\author{
Ineke Pillet, Hans Op de Beeck and Haemy Lee Masson*
}

Laboratory of Biological Psychology, Department of Brain and Cognition, Leuven Brain Institute, KU Leuven, Leuven, Belgium

The invention of representational similarity analysis [RSA, following multi-voxel pattern analysis (MVPA)] has allowed cognitive neuroscientists to identify the representational structure of multiple brain regions, moving beyond functional localization. By comparing these structures, cognitive neuroscientists can characterize how brain areas form functional networks. Univariate analysis (UNIVAR) and functional connectivity analysis (FCA) are two other popular methods to identify functional networks. Despite their

OPEN ACCESS

Edited by:

Amir Shmuel,

McGill University, Canada

Reviewed by:

Mete Ozay,

PKSHA Technology, Japan

LiSu,

University of Cambridge,

United Kingdom

*Correspondence:

Haemy Lee Masson

haemy.leemasson@kuleuven.be

Specialty section:

This article was submitted to

Brain Imaging Methods,

a section of the journal

Frontiers in Neuroscience

Received: 05 April 2019

Accepted: 02 December 2019

Published: 08 January 2020

Citation:

Pillet I, Op de Beeck H and Lee Masson H (2020) A Comparison of Functional Networks Derived From Representational Similarity, Functional Connectivity, and Univariate Analyses.

Front. Neurosci. 13:1348 doi: 10.3389/fnins.2019.01348 popularity, few studies have examined the relationship between networks from RSA with those from UNIVAR and FCA. Thus, the aim of the current study is to examine the similarities between neural networks derived from RSA with those from UNIVAR and FCA to explore how these methods relate to each other. We analyzed the data of a previously published study with the three methods and compared the results by performing (partial) correlation and multiple regression analysis. Our findings reveal that neural networks resulting from RSA, UNIVAR, and FCA methods are highly similar to each other even after ruling out the effect of anatomical proximity between the network nodes. Nevertheless, the neural network from each method shows unique organization that cannot be explained by any of the other methods. Thus, we conclude that the RSA, UNIVAR and FCA methods provide similar but not identical information on how brain regions are organized in functional networks.

Keywords: fMRI, multi-voxel pattern analysis (MVPA), representational similarity analysis (RSA), univariate analysis, functional connectivity (FC)

\section{INTRODUCTION}

Multi-voxel pattern analysis (MVPA) has recently become one of the most frequently used techniques for analyzing fMRI data. It considers the spatial pattern of neural activation across multiple voxels and examines whether these patterns contain task-related information (Haxby et al., 2001; Haynes and Rees, 2006; Mur et al., 2009; Kriegeskorte, 2011; Haxby, 2012; Coutanche, 2013; Haynes, 2015). It is referred to as multivariate

Abbreviations: FCA, functional connectivity analysis; MVPA, multi-voxel pattern analysis; RSA, representational similarity analysis; UNIVAR, univariate analysis. 
or multi-voxel because it analyzes a set of voxels together (the pattern of activation of this set) instead of modeling activity of a single voxel (as is done in univariate analysis) (Norman et al., 2006; Mur et al., 2009; Kriegeskorte, 2011; Yang et al., 2012). In addition, patterns of activation can be used to investigate the similarities between such patterns of different conditions, for example the similarity of the activation pattern when viewing a face vs. when viewing a scene, or between such activation patterns of different brain regions in a certain condition (Mur et al., 2009; Haxby, 2012). This approach is referred to as representational similarity analysis (RSA) (Kriegeskorte et al., 2008). In first-order RSA, a representational dissimilarity matrix (RDM) is set up to understand the dissimilarity between patterns of activation of different stimuli in a certain brain region (Kriegeskorte et al., 2008; Yang et al., 2012). In second-order RSA, RDMs are compared between brain regions (Kriegeskorte et al., 2008; Yang et al., 2012). This method has been referred to as representational connectivity as it allows to identify the representational relationship among brain regions (Kriegeskorte et al., 2008). Connectivity related to multivariate information has since then been given a more specific meaning to refer to analyses of the temporal dynamics of the information contained in multi-voxel patterns, also sometimes referred to as multivariate or informational connectivity (Coutanche and Thompson-Schill, 2014; Anzellotti and Coutanche, 2018). For this reason, we opted for the more general RSA term instead of using the term representational connectivity.

Univariate analysis (UNIVAR) and functional connectivity analysis (FCA) are two other frequently used techniques for analyzing fMRI data. UNIVAR assesses neural activation of an individual voxel or a mean activation across voxels of a brain region. For this reason, it is often used to localize brain regions engaged in processing a particular type of stimuli (e.g., face vs. object) and thereby draw conclusions about the regions that are involved in cognitive processes important for the stimuli or task at hand (Haynes and Rees, 2006; Logothetis, 2008; Mur et al., 2009; Coutanche, 2013; Haynes, 2015). It is referred to as univariate because a general linear model (GLM) is applied voxelwise to relate the experimental design to the neural activity of each voxel's time-course in the brain (Raizada and Kriegeskorte, 2010). FCA (for a review, see Friston, 2011) characterizes the communication between brain regions during rest or a task (Friston, 1994), measuring the strength of the relation between BOLD time-series signals of brain regions (Yang et al., 2012; Geerligs et al., 2016). When FCA is applied to a resting-state fMRI dataset, it reveals the intrinsic network of the brain based on low-frequency BOLD fluctuations of brain regions (Biswal et al., 1995; Cordes et al., 2001; Fox and Raichle, 2007). This intrinsic network can also be extracted from a task-based fMRI dataset by removing the task-induced signal from the data (Fair et al., 2007). Therefore, this method is often referred to as intrinsic functional connectivity.

Notably, there are various conceptual similarities and differences between RSA, UNIVAR, and FCA. UNIVAR and FCA methods are similar in that they average across the BOLD signal of all the voxels in a brain region, unlike MVPA (see Figure 1). The organization of networks from co-activation has also proven to be similar to those from resting-state connectivity (Crossley et al., 2013). Analogously, Anzellotti and Coutanche referred to this type of FCA as univariate FCA (Anzellotti and Coutanche, 2018). Second-order RSA and FCA are similar in that they are both based on a measure of the similarity between brain regions. When using correlations, correlating the averaged BOLD timeseries signals between the regions of interest (ROIs) in FCA is methodologically similar to correlating RDMs of those ROIs in second-order RSA (Xue et al., 2013). UNIVAR and RSA, or at least MVPA, have been frequently compared when describing functional properties of one region of the brain (e.g., see Jimura and Poldrack, 2012; Coutanche, 2013; Davis et al., 2014; Gilron et al., 2017). A significant finding from these studies was that changes (across different stimuli) in the activation patterns could be detected even when conditions were not different in the average univariate activation in a region (Mur et al., 2009). For example, different speech sounds showed different activation patterns in the right auditory cortex, but the average activation of this region across those speech sounds did not differ (Raizada et al., 2010). These studies have provided valuable insights into the conceptual and empirical relationships between UNIVAR and MVPA. Similarly, studies have used both RSA and FCA, some drawing the same conclusion from the results of RSA and FCA (e.g., Zeharia et al., 2015), or not (Boets et al., 2013; Bulthé et al., 2018).

A study that directly and simultaneously compares networks resulting from RSA with those from UNIVAR and FCA is missing from today's literature. Thus, the current study explores how the networks from RSA complement the networks from UNIVAR and FCA when investigating the functional architecture of the brain. Although direct and simultaneous comparisons between brain networks based on UNIVAR, RSA and FCA have not been performed (to our knowledge), we expect at least some convergence. For example, we hypothesize that brain regions with similar representational similarity would tend to be functionally connected, without excluding the possibility of uniqueness in the networks resulting from the two methods. Specifically, given the evidence of the topographic arrangement of the basic sensory cortical areas, such as the visual and sensorimotor cortex (see Kaas, 1997, for a detailed review) and given that these low-level visual or sensorimotor areas respectively are similar in what they do even at rest (Cordes et al., 2000), we predict that the way in which brain networks composed of visual or sensorimotor areas are constructed, would be highly similar in all three methods. More precisely, we predict that the visual network organization and the sensorimotor network organization respectively, as obtained by UNIVAR, RSA, and FCA, will be highly similar. In sum, the goal of this study is to explore how the networks derived from RSA compare to those from UNIVAR and FCA. To answer this question, we applied second-order RSA to a previously reported fMRI study (Lee Masson et al., 2018) and compared the resulting networks with the results obtained from UNIVAR and intrinsic FCA. In particular, we conducted (partial) correlation and multiple regression analysis (comparing them to signal-to-noise ratio measurements), controlling for the confounding influence of anatomical proximity between 


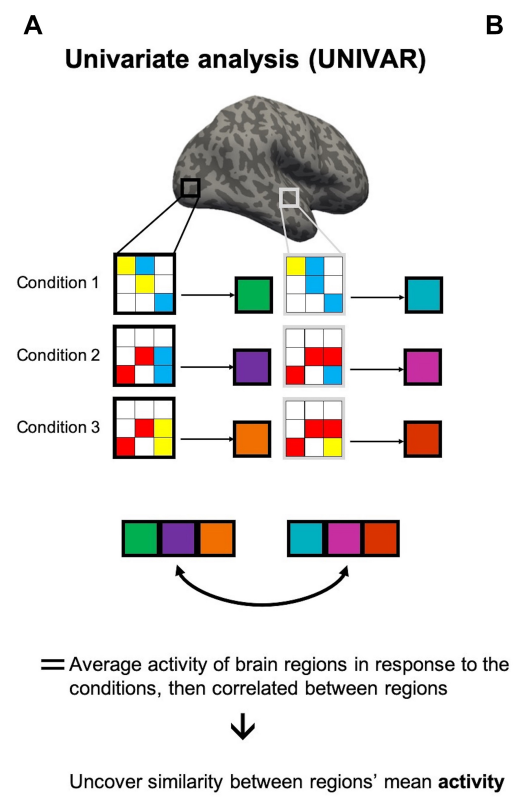

B
Representational similarity analysis (RSA)

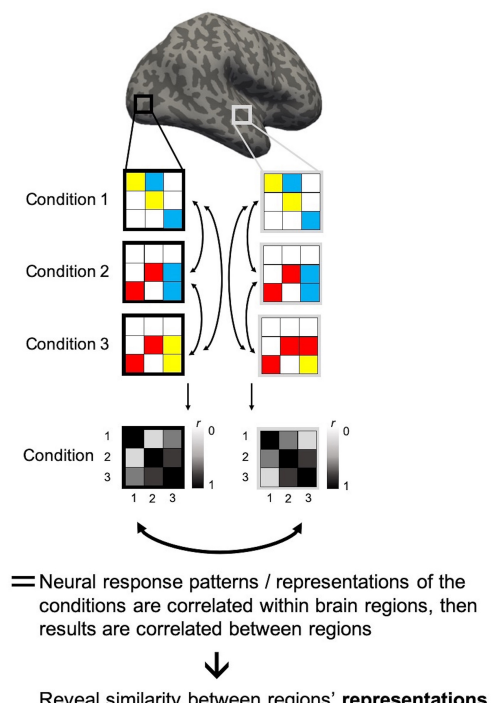

Reveal similarity between regions' representations

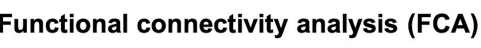

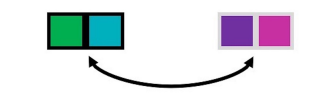

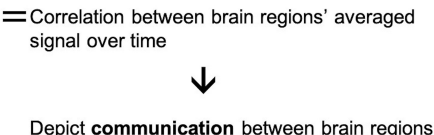

FIGURE 1 | Representational similarity, univariate and functional connectivity analyses. Visualization of the several steps of the methodologies of these analyses to clarify the (dis)similarities. Gray and white squares drawn on the brain depict two random regions of interest (ROls), consisting of 9 voxels each. Within these squares, colors in the $3 \times 3$ matrix depict the BOLD signal intensity of the voxels in a condition 1, 2, or 3, modeled through a general linear model (GLM). High intensity is shown in red, medium intensity in yellow, low intensity in blue. (A) In univariate analyses (UNIVAR), the signal is averaged across all voxels within the ROI, and this for every ROI and every condition. This step is visualized by large squares next to each matrix with a color depicting the calculated average. Consequently, these results are averaged across participants. To understand if the ROls are similarly activated in light of the conditions, the results of every ROI can be correlated to each other. (B) In representational similarity analysis (RSA), the signal is not averaged across voxels within ROls. Instead, the signal of an array of voxels in a ROI in light of a certain condition is correlated to that of another condition. All possible pairwise comparisons are made. This step is depicted by a square matrix containing the correlation results for each condition pair: the color of each element of the matrix indicates the strength of the correlation (black indicates a correlation of 1 and white indicates 0). These results show if conditions are represented similarly within a ROI. They are then averaged across subjects. Then, these results can be correlated between ROls to understand if different ROls represent the conditions in a similar way. (C) In functional connectivity analysis, the signal of the voxels in a $\mathrm{ROI}$ at different time-points (i.e., change in BOLD signal intensity over time for each voxel) is averaged across all voxels within that ROI. This step is visualized by large squares with a color depicting the calculated average. After, the resulting average BOLD signal time course can be correlated between ROIs and consequently averaged across subjects. This analysis method depicts if two ROls communicate.

brain regions of interest on RSA, UNIVAR, and intrinsic FCA results. In addition, we explored our results visually by implementing multi-dimensional scaling (MDS) and Procrustes transformation methods.

\section{MATERIALS AND METHODS}

\section{Datasets}

We reanalyzed data from our previous fMRI study (Lee Masson et al., 2018). All participants provided written informed consent before the experiment in accordance with the Declaration of Helsinki. The study was carried out in accordance with the recommendations of and approved by the Medical Ethical Committee of KU Leuven (S53768 and S59577). In this study, 21 healthy participants observed grayscale videos (see Figure 2) of social touch interaction, varying in valence and arousal (Lee Masson and Op de Beeck, 2018). The experiment included 39 social touch videos, and in addition 36 non-social control videos. Participants carried out an orthogonal attention task: they pressed a button with their left or right thumb whenever the touch interaction initiator wore a gray or black shirt, depending on the instruction of that specific run (left for gray, right for black). The stimuli were displayed for $3 \mathrm{~s}$, followed by an inter-stimulus interval of $3 \mathrm{~s}$ during which a fixation cross was presented and during which the participants could press a button as a response related to the task. Each run was divided into three blocks of 25 videos. At the start of each block, a baseline (display of a fixation cross) of $6 \mathrm{~s}$ was included. The total duration of each run was $7.80 \mathrm{~min}$. The participants completed six runs. In the following UNIVAR and RSA analyses, we restrict the analyses to the data from the 39 social touch videos.

Importantly, when creating the videos, we controlled for the visual elements, such as clothes style and color of the actors so that these do not induce a visually biased neural response (Lee Masson and Op de Beeck, 2018). For example, having actors wear blue in the pleasant touch scenes and having actors wear red in unpleasant touch scenes can induce visual bias related to the clothing color when contrasting the brain response between the pleasant and unpleasant touch conditions.

In addition, the scan sessions included runs in which participants received (instead of observing) pleasant (brush strokes) and unpleasant touch (rubber band snaps) in a block 


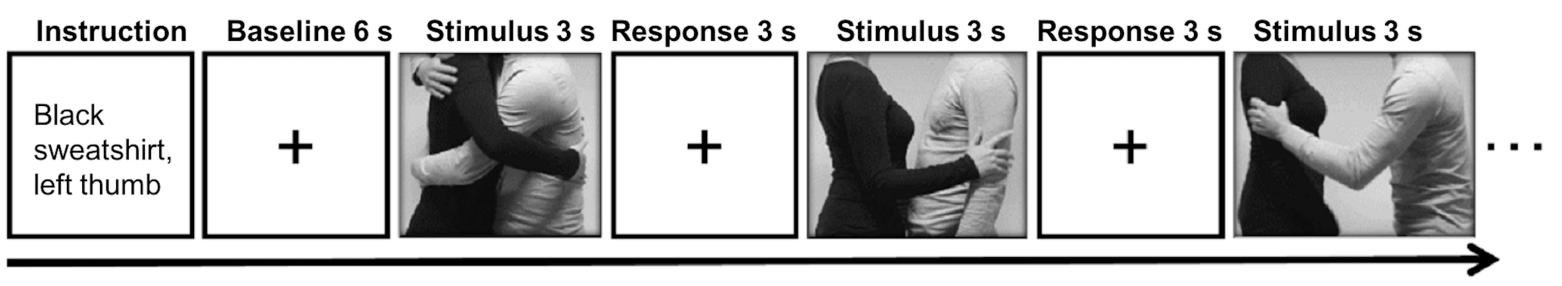

FIGURE 2 | The experimental procedure. Participants received instruction on when they should press a certain button (e.g., press the button with your left thumb when touch interaction initiator wears black sweatshirt). After a baseline of $6 \mathrm{~s}$, the stimuli were presented for $3 \mathrm{~s}$ always followed by an inter-stimulus interval of $3 \mathrm{~s}$, during which a fixation cross was presented and participants could press a button. In this example, still frames of three social touch videos are shown (left: hug, middle: stroke, right: shake). All videos can be found here: https://osf.io/ng5mf/.
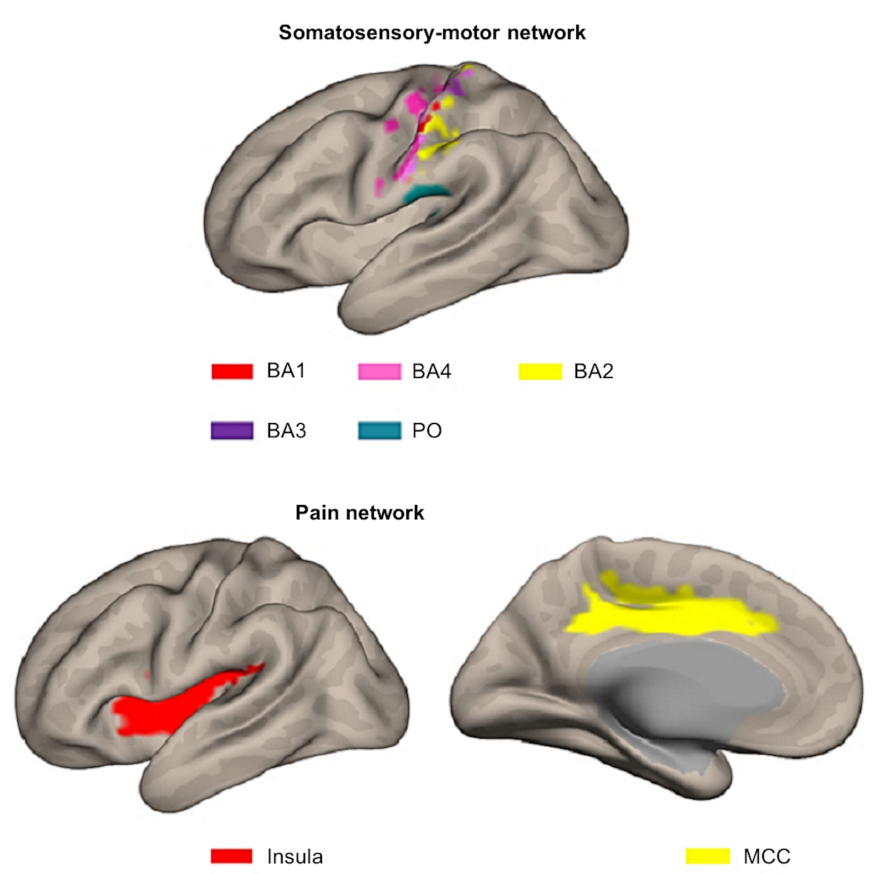
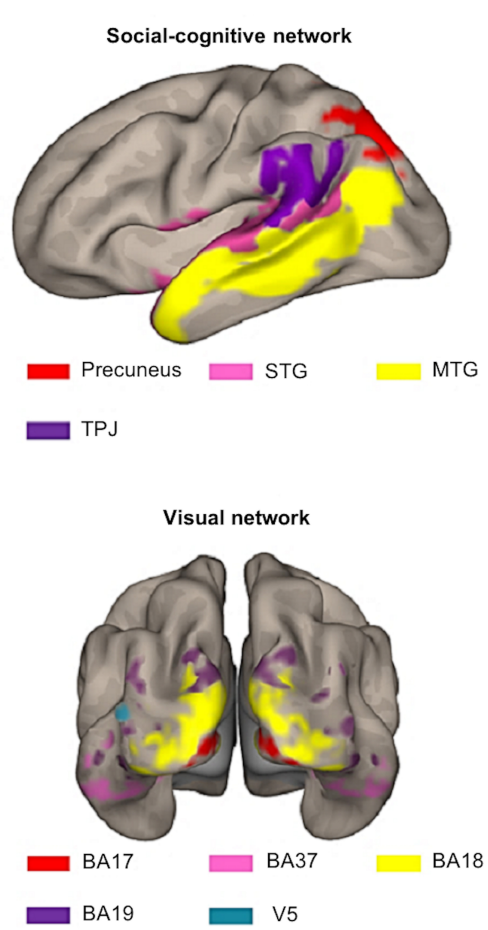

FIGURE 3 | Illustration of the different ROls in the context of the network they were assigned to a priori. Top left: somatosensory-motor network ROls including BA1 (red), BA4 (pink), BA2 (yellow), BA3 (purple), and PO (blue). Top right: social-cognitive network ROls including precuneus (red), STG (pink), MTG (yellow), and TPJ (purple). Bottom left: pain network ROls including insula (red) and MCC (yellow). Bottom right: visual network ROls including BA17 (red), BA37 (pink), BA18 (yellow), BA19 (purple), and V5 (blue). This figure was made using CONN toolbox 17 (Whitfield-Gabrieli and Nieto-Castanon, 2012).

design (see Lee Masson et al., 2018, for more details). These data were used for the intrinsic FCA.

\section{Regions of Interest (ROIs)}

For our previous study (Lee Masson et al., 2018), we selected 16 a priori defined ROIs, belonging to four different networks in the brain that proved to be important in processing observed social touch interactions: the somatosensory-motor network [the parietal operculum (PO), Brodmann area (BA) 3, BA1, BA2, BA4 (Rolls et al., 2003)], the social-cognitive network [the middle temporal gyrus (MTG), the precuneus, the superior temporal gyrus (STG), the temporoparietal junction (TPJ) (Jacoby et al., 2016)], the pain network [the middle cingulate cortex (MCC), the insula (Gordon et al., 2013; Lamm and Majdandžić, 2015;
Morrison et al., 2011)], and the visual network given that visual stimuli were used (BA17, BA18, BA19, BA37, V5) (see Figure 3). To define these ROIs anatomically, first, we made masks with various templates from PickAtlas software (Maldjian et al., 2003, RRID:SCR_007378), SPM Anatomy toolbox (Eickhoff et al., 2005, RRID:SCR_013273) and connectivity-based parcellation atlas (Mars et al., 2012). Second, we extracted all the voxels in the mask per ROI and combined left and right hemispheres. Afterward, we examined if there were overlapping voxels among ROIs (e.g., V5 is located in BA19 and BA37) and removed overlapping voxels from each other in order to ensure all ROIs are anatomically independent. For further information about these ROIs and how they were defined, see our previous study (Lee Masson et al., 2018). In contrast to FCA that 
often includes a more extensive set of ROIs, the RSA method requires ROIs to contain meaningful neural signals associated with the experimental conditions. For this reason, only the aforementioned 16 ROIs, whose spatial neural patterns passed the MVPA reliability test, were selected (Lee Masson et al., 2018). Briefly, in this reliability test, runs are split into two halves and the correlation between neural patterns for withinand between-conditions are compared per ROI. This process is repeated 100 times (to randomly split the runs into two halves) and these results are then averaged. ROIs are only included if the correlations for within-condition comparisons are significantly stronger than those for between-condition comparisons. Neural pattern similarity between different conditions most likely only reflects noise when neural pattern similarity between the same conditions is low (Ritchie et al., 2017; for more details on this test and the results; see Lee Masson et al., 2018).

\section{Univariate Analysis}

In our previous study, we processed functional data by using a standard preprocessing pipeline and by applying a general linear model (GLM) to each subject's data (Lee Masson et al., 2018). On top of the regressors of interest (matched to the onset time of each regressor (duration $=0$ ) of the event-related design of the fMRI observing touch experiment), six head motion parameters were included in the models as nuisance covariates (Lee Masson et al., 2018). These GLMs were defined with data smoothed with $8 \mathrm{~mm}$ FWHM. For detailed information on how data was preprocessed and how the GLM was applied, see our previous study (Lee Masson et al., 2018). From these GLMs, we obtained the estimated beta-values per stimulus of the social condition $(N=39)$ for all voxels in each ROI. For each stimulus, we averaged the beta values of all runs, of all voxels within each ROI, and of all participants, yielding a one-dimensional array with 39 elements in each ROI, reflecting how strongly each of the 39 videos activated the ROI. These arrays were Pearson correlated for each possible pair combination of ROIs to investigate the similarity between ROIs' average BOLD responses evoked during the observation of social touch and therefore to investigate clustering/networks of our ROIs with regard to their average activations. We refer to this clustering as the activation network.

\section{Representational Similarity Analysis}

This analysis was based upon a GLM applied to the fMRI observing touch experiment that consisted of 75 predictors (one for each video). The preprocessing pipeline for this analysis differed slightly from the one used for univariate analysis: data was smoothed at $5 \mathrm{~mm}$ FWHM. As such, we optimized the preprocessing parameters to fit the requirements of each analysis (Hendriks et al., 2017). For each ROI, we created a $39 \times 39$ neural matrix by correlating (Pearson) the multi-voxel patterns between all possible combinations of pairs of stimuli of the social condition $(N=39)$ and then averaged this across subjects (first-order RSA, Kriegeskorte et al., 2008). After, we vectorized the upper diagonal elements of this group-averaged matrix while discarding the diagonal and lower diagonal elements, and correlated (Pearson) these vectors for all possible combinations of ROI pairs (second-order RSA, Kriegeskorte et al., 2008).
These comparisons between areas allow us to investigate the representational similarity between ROIs and therefore to investigate clustering/networks of our ROIs with regard to the between-condition similarity in multi-voxel activation patterns (Kriegeskorte et al., 2008). We refer to this clustering as the representation network. More information on the details of how MVPA was applied to fMRI data can be found in our previous study (Lee Masson et al., 2018).

\section{Functional Connectivity Analysis}

Functional connectivity analysis, performed in the CONN toolbox 17 (Whitfield-Gabrieli and Nieto-Castanon, 2012, RRID:SCR_009550), was applied to a different set of fMRI data (wherein participants received touch) obtained in the same scan sessions. We used two independent sets of fMRI data to avoid a spurious correlation between the two sets of brain networks resulting from UNIVAR and FCA. BOLD signal fluctuations may be partially induced by the presented stimuli, which may result in shared signals between networks derived from the UNIVAR and FCA methods. In the other direction, spontaneous fluctuations over time may affect the estimated univariate activation.

Preprocessing was conducted as described in our previous study (Lee Masson et al., 2018), but again optimized to fit the requirements of FCA: no smoothing was carried out to avoid a spillover effect (Alakörkkö et al., 2017). The outlier scans were detected based on the global signal spike and motion in the functional data by the Artifact Detection Toolbox (ART) software package $^{1}$. Consequently, standard denoising methods were applied to remove confounding effects. This step consists of (1) linearly regressing out 13 principal components of white matter and cerebrospinal fluid signals, six head motion parameters and their first-order derivatives, all scrubbing covariates from the artifact detection, and main task effects (rest condition, see below), (2) linear detrending, and (3) band-pass filtering $(0.008-0.09 \mathrm{~Hz})$ that removes slowly fluctuating noise, such as scanner drift, and the task-induced signal. To calculate intrinsic FC (functional connectivity), we did not encode task-related information in the experimental design. Instead, task effect (i.e., receiving touch) was removed from the fMRI time series by including regressors corresponding to each task condition during the denoising step, and the rest condition was defined (Fair et al., 2007). Previous studies indicated that the intrinsic fluctuations in a BOLD signal would only be weakly affected by task demands and could be separated when entangled with the task-related signals (Fox et al., 2006; Fair et al., 2007). Several studies have implemented this approach on task-based fMRI data to yield the intrinsic functional connectivity network (e.g., Fair et al., 2007; Bassett et al., 2011; Boets et al., 2013; Ebisch et al., 2013).

For each subject, a GLM was performed to assess bivariate Pearson correlation coefficients between ROIs' BOLD timeseries. These coefficients were averaged across subjects. As a result, networks of functionally connected (communicating) regions were uncovered. We refer to this clustering as the connectivity network.

\footnotetext{
${ }^{1}$ www.nitrc.org/projects/artifact_detect/
} 


\section{Signal-to-Noise Ratio Measurement}

To measure the reliability of the fMRI signal for the activation (from UNIVAR), representation (from RSA) and connectivity (from FCA) network, we randomly split the participants into two groups ( $n=10$ or 11 per group). For each of these analyses, we correlated the resulting activation, representation, and connectivity network matrices (off-diagonal values) of one group with the matrix of the other group. This process was performed for a total of 100 iterations (each time randomly splitting the data into two groups). The correlations were adjusted with the Spearman-Brown split-half reliability formula and then averaged (across the 100 iterations) for UNIVAR, RSA, and FCA separately. The results from the between-subject correlations work as a measure of signal-to-noise ratio (SNR), taking the between-subject variability in the neural data into account, in that it estimates the maximum correlation we could expect. The correlation between the same types of data from the two subgroups (group 1 vs. group 2 in FCA results) should be higher than the correlation with another type of data (e.g., FCA vs. RSA results). This SNR correlation coefficient was also squared to obtain the proportion of the variance in the signal that can be explained by other variables.

\section{Anatomical Proximity}

For each ROI per hemisphere, we collected the $x-y-z$ coordinates of its voxels. Consequently, for each ROI pair, we calculated Euclidean distances for all possible pairs of voxels between these two ROIs. Among these calculated distances, we use the minimum value per ROI pair as a measure of the anatomical distance between the two ROIs. Then, we averaged the distances across the two hemispheres. We also performed supplementary analyses with distance based on the average rather than the minimum value, which yielded very similar results (the two indices correlate strongly, correlation $r=0.81$ ). As a final step, we inverted these results to have a measure of anatomical proximity instead of distance with the maximal distance becoming the minimal proximity zero. We refer to these results as the anatomical proximity network. Dependency of functional connectivity on anatomical distance has been observed (Salvador et al., 2005). Thus, the anatomical proximity network was included in the partial correlation and the multiple regression model to rule out the effects of anatomical proximity when comparing the activation, representation and connectivity network.

\section{Comparing the Activation, Representation and Connectivity Network \\ (Partial) Correlation Models}

To understand how similar the activation, representation, connectivity and anatomical proximity network are, we conducted a rank-order correlational analysis between these networks. In addition, we also computed the partial Spearman correlation coefficient to understand the similarities between the two networks while controlling for the remaining networks. To draw statistical inferences, we conducted a permutation test, wherein one of the variables of interest [one of the networks, consisting of all possible unique ROI pairs (120 pairs)] was randomly shuffled and then (partially) correlated with the unshuffled variables [remaining original networks, each consisting of all possible unique ROI pairs (120 pairs per network)]. This process was iterated a 1000 times. These permutation tests provide empirical $p$-values (probability values) reflecting the proportion of permutations wherein the (partial) correlations with the shuffled data were larger (or equally large) than the original (partial) correlations.

\section{Multiple Regression Models}

Following up on the (partial) correlation models, we conducted multiple regression analysis to investigate if the activation, representation or connectivity network respectively, could be explained by the other remaining networks. The anatomical proximity network was also included in all of the multiple regression models. Z-score standardizations were performed to normalize the data before building a regression equation. Similarly to the correlational analysis, permutation tests were used to obtain empirical $p$-values. In the end, the percentage variance explained by the model was compared to the squared signal-to-noise ratio of the predicted variables of the model.

\section{Multi-Dimensional Scaling (MDS) and Procrustes Transformations}

We conducted multi-dimensional scaling (MDS) on the activation, representation, and connectivity network matrices to visualize the networks in a two-dimensional space that shows the distance between each pair of ROIs based on how dissimilar these ROIs are in terms of their activation, representation, and connectivity respectively. We used the built-in MATLAB function "mdscale" with default parameters, minimizing the default goodness-of-fit criterion "stress" and 100 replicates of the scaling. MDS results of the representation network were used as a template to which the MDS results of the activation and connectivity networks were aligned using Procrustes transformations, to visualize the networks in the same space. For this, we used the built-in MATLAB function "procrustes" with default parameters.

\section{RESULTS}

\section{Networks}

In total, we have four matrices (see Figure 4). For three of the methods (RSA, UNIVAR, and FCA) the values in the matrices are based upon correlational analyses. In each of these matrices, we had a large range of values. For the representation network, for which vectorized first-order RSA results were correlated between all ROI pairs, correlations range from 0.07 (V5 insula) to 0.82 (BA3 - BA4). In the activation network matrix, the correlation results range from -0.01 (precuneus - $\mathrm{PO}$ ) to 0.98 (BA3-BA4). The values of the ROI-to-ROI connectivity range from -0.17 (precuneus - $\mathrm{PO}$ ) to 0.83 (BA3-BA4). The anatomical proximity network values range from 0 to 67.63 . The higher the value, the more closely the two ROIs are located. 

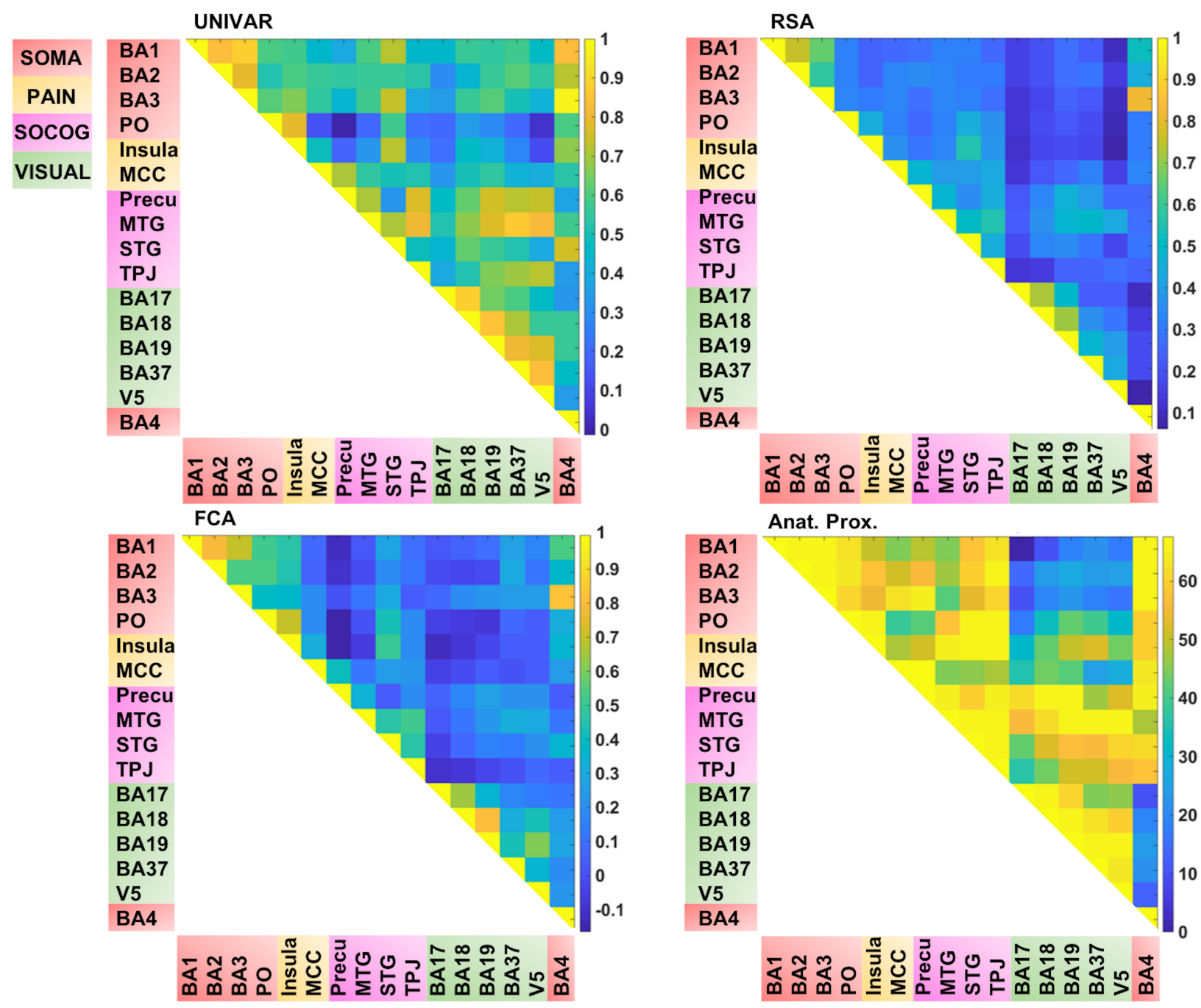

\begin{tabular}{l|}
\hline BA1 \\
BA2 \\
BA3 \\
P0 \\
Insula \\
MCC \\
Precu \\
MTG \\
STG \\
TPJ \\
BA17 \\
BA18 \\
BA19 \\
BA37 \\
V5 \\
BA4
\end{tabular}

FIGURE 4 | Visualization of the different networks before z-score standardization of the correlation coefficients. Top left: activation network (UNIVAR), top right: representation network (RSA), bottom left: connectivity network (FCA), bottom right: anatomical proximity network. Yellow in the matrix = when two ROls are very similar in their activation (UNIVAR results) or their representation (RSA results), are well connected (FCA results), or are located closely in the brain. Blue in the matrix = when two ROls are very different in their activation (UNIVAR results) or their representation (RSA results), are not connected (FCA results), or are located remotely in the brain. SOMA (red), somatosensory-motor network areas; PAIN (yellow), pain network areas; SOCOG (purple), social-cognitive network areas; VISUAL (green), visual network areas.

As the values are inverted distances, a value of 0 indicates the minimum anatomical proximity between ROIs (e.g., BA1BA17), which in the original distance was $67.63 \mathrm{~mm}$. A proximity value of 67.63 indicates the maximum anatomical proximity between ROIs: these ROIs are located right next to each other (e.g., BA1-BA2).

Each of the matrices was very reliable. The signal-tonoise ratio estimated from the results of between-subjects correlations was $r=0.92$ (squared to obtain explainable variance: $E V=85 \%$ ) for the representation network, $r=0.96$ $(E V=92 \%)$ for the activation network, and $r=0.97(E V=94 \%)$ for the connectivity network. As illustrated in Figure 4, the representation, the activation, and connectivity networks look highly similar to each other. For example, the high correlation values between the ROIs in somatosensory areas such as BA3, BA1, and BA2 are apparent in all of these networks: these ROIs contain similar task-related information (based on RSA results), are activated to a similar level (based on UNIVAR results) and are functionally linked to each other (based on FCA results). BA4, the motor area, is strongly correlated to BA3 and BA1 in the representation, activation, and connectivity network, but only moderately to BA2. Another example is the moderate to high correlation between visual areas, found in the representation, activation and connectivity network. In sum, this finding applies to all four ROI networks. Areas of different ROI networks typically show lower correlations, which is again consistent across methods. For example, the moderate correlations between social-cognitive areas and visual 
areas can be found in the representation, activation and connectivity network.

\section{Comparing Networks}

To understand the (dis)similarity between representation, activation, connectivity, and anatomical proximity networks more quantitatively, we tested the linear relationship among these networks. The results indicated that all networks are similarly organized in the context of brain function and anatomy, with the Spearman rank-order correlations (all significant) ranging from 0.53 to 0.79 (see Table 1). In addition, the partial correlation coefficients were computed between two networks after removing the effect of the other remaining networks. The results from partial correlation (including all four networks) demonstrated that, after controlling for the other networks, the activation and connectivity network (Spearman correlation $r_{S}=0.50$, $p<0.001)$, the representation and activation network $\left(r_{S}=0.34\right.$, $p<0.001)$, and the representation and anatomical proximity network $\left(r_{S}=0.67, p<0.001\right)$ still correlate significantly (see Table 2). Conversely, the measured partial correlation between the representation and connectivity network was no longer significant after ruling out the effects of the other covariates (partial $r_{S}=0.10$ ), implying that their association is fully explained by their relationship with other networks. The partial correlation between the connectivity and anatomical proximity network (partial $r_{S}=0.15$ ), and between the activation and anatomical proximity network (partial $r_{S}=0.06$ ) was also no longer significant.

As an alternative approach, we also implemented multiple regression models. Similar to the (partial) correlation measurements, these regression models quantify the relations between the networks, but in addition the regression models provide an estimate of the total variance in a network that can be explained by all other networks.

TABLE 1 | The correlations between the activation (UNIVAR), representation (RSA), connectivity (FCA) and anatomical proximity (Anat. Prox.) network.

\begin{tabular}{lcccc}
\hline & UNIVAR & RSA & FCA & Anat. Prox. \\
\hline UNIVAR & 1 & $0.66^{*}$ & $0.70^{*}$ & $0.53^{*}$ \\
RSA & $0.66^{*}$ & 1 & $0.61^{*}$ & $0.79^{*}$ \\
FCA & $0.70^{*}$ & $0.61^{*}$ & 1 & $0.55^{*}$ \\
Anat. Prox. & $0.53^{*}$ & $0.79^{*}$ & $0.55^{*}$ & 1 \\
\hline
\end{tabular}

*Significant correlation.

TABLE 2 | The partial correlations between the activation (UNIVAR), representation (RSA), connectivity (FCA) and anatomical proximity (Anat. Prox.) network.

\begin{tabular}{lcccc}
\hline & UNIVAR & RSA & FCA & Anat. Prox. \\
\hline UNIVAR & 1 & $0.34^{*}$ & $0.50^{*}$ & -0.10 \\
RSA & $0.34^{*}$ & 1 & 0.10 & $0.67^{*}$ \\
FCA & $0.50^{*}$ & 0.10 & 1 & 0.15 \\
Anat. Prox. & -0.10 & $0.67^{*}$ & 0.15 & 1 \\
\hline
\end{tabular}

*Significant partial correlation.
A first model tested if the connectivity, activation and anatomical proximity network significantly predicted the representation network. The coefficient of determination from the regression equation indicated that these three predictors explained $71.2 \%$ of variability in the representation network $\left[R^{2}=0.712, F(3,116)=96, p<0.001\right]$. The squared signalto-noise ratio (based on the between-subjects correlation) in the representation network indicated $85 \%$ of the variance to be explainable, leaving approximately $14 \%$ of the signal unexplained. In addition, we calculated the $\beta$ coefficients to examine the degree to which each predictor independently contributes to the prediction of the representation network. According to the results, the anatomical proximity network significantly contributed to the prediction of the representation network $(\beta=0.40, p<0.001)$, as did the connectivity network $(\beta=0.36$ $p=0.004)$ and the activation network $(\beta=0.26, p=0.03)$.

Similarly, we predicted the connectivity network based on the representation, activation, and anatomical proximity network, using multiple regression analysis. The results indicated that the predictors explained $59.6 \%$ of variability in the connectivity network $\left[R^{2}=0.596, F(3,116)=57, p<0.001\right]$. The squared signal-to-noise ratio (based on the between-subjects correlation) in the connectivity network indicated $94 \%$ of the variance to be explainable, leaving approximately $34 \%$ of the signal unexplained. When examining the independent contributions of each predictor, we found out that the representation network significantly contributed to the prediction of the connectivity network $(\beta=0.51, p=0.003)$, as did the activation network $(\beta=0.37, p=0.005)$, but not the anatomical proximity network $(\beta=-0.07, p=0.55)$.

Lastly, we tested if the representation, connectivity, and anatomical proximity network significantly predicted the activation network. The results revealed that the predictors explained $55.8 \%$ of variability in the activation network $\left[R^{2}=0.558, F(3,116)=49, p<0.001\right]$. The squared signalto-noise ratio (based on the between-subjects correlation) in the activation network indicated $92 \%$ of the variance to be explainable, leaving approximately $36 \%$ of the signal unexplained. The predictors indicated that the representation network significantly contributed to the prediction of the activation network $(\beta=0.41, p=0.01)$, as did the connectivity network $(\beta=0.41, p=0.001)$, but not the anatomical proximity network $(\beta=-0.01, p=0.91)$.

Thus, for each type of network, we find that a lot of the organization can be predicted from the other networks, but there is also some remaining variance left unexplained. We visualized this unique signal left in each of these networks after regressing out the signal explained by the other networks from the representation, activation and connectivity network respectively (see Figure 5B). In Figure 5B, in contrast to Figures $\mathbf{4}, \mathbf{5 A}$ (which takes the values of Figure 4 and $\mathrm{z}$-score standardizes them, for reasons mentioned above), the networks now do not look similar: they show different patterns.

Several unique findings concerning correlations between ROInetworks can be observed in Figure 5B. For example, socialcognitive brain areas correlate strongly to other visual areas in the 


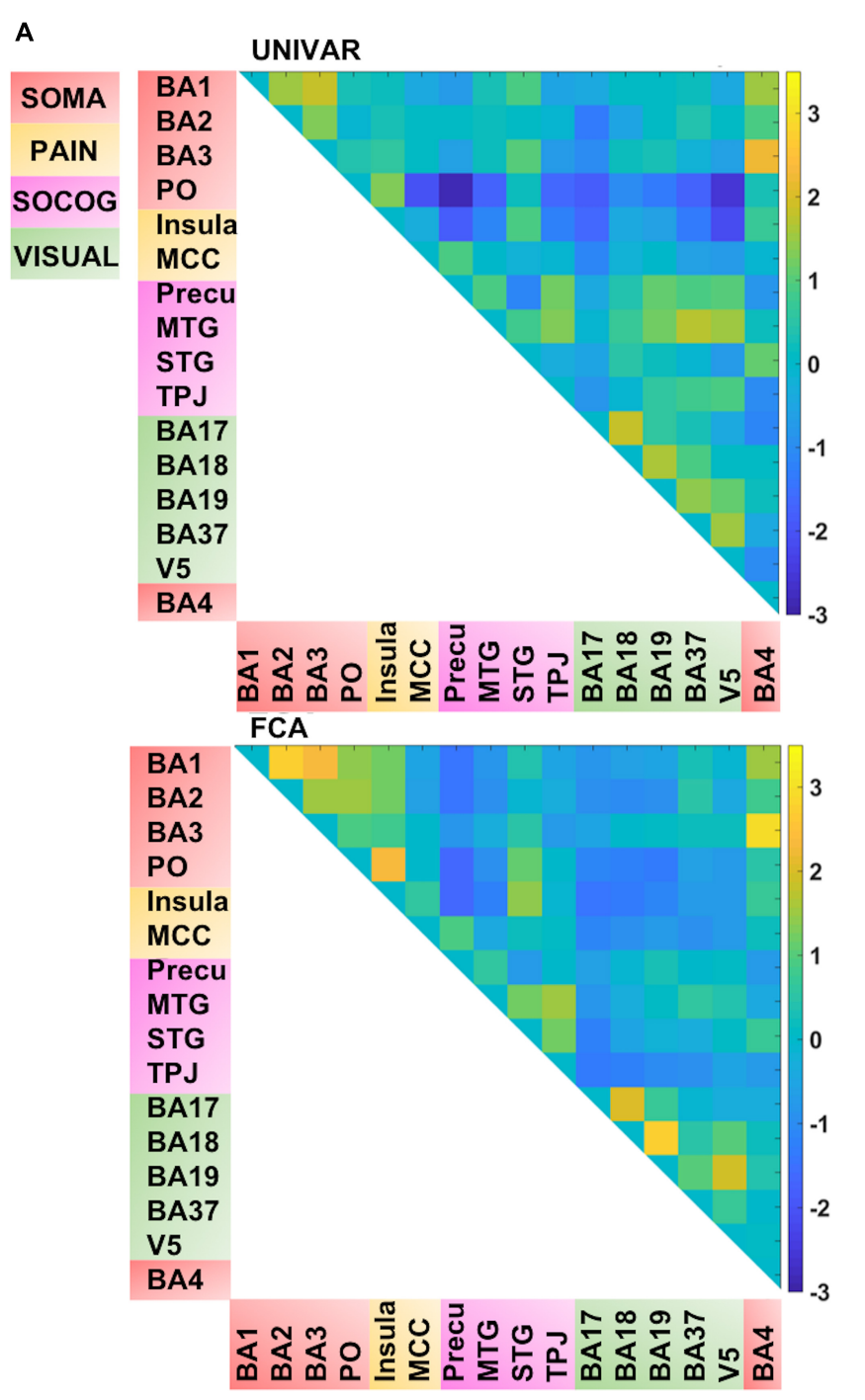

FIGURE 5A | Visualization of the different networks after z-score standardization of the correlation coefficients. Top left: activation network (UNIVAR), top right: representation network (RSA), bottom left: connectivity network (FCA), bottom right: anatomical proximity network. Yellow in the matrix = when two ROls are very similar in their activation (UNIVAR results) or their representation (RSA results), are well connected (FCA results), or are located closely in the brain. Blue in the matrix = when two ROls are very different in their activation (UNIVAR results) or their representation (RSA results), are not connected (FCA results), or are located remotely in the brain. SOMA (red), somatosensory-motor network areas; PAIN (yellow), pain network areas; SOCOG (purple), social-cognitive network areas; VISUAL (green), visual network areas.

activation network (e.g., $r$ (before z-score standardization) $=0.69$ between TPJ and BA37) while this is moderate to low in the representation (e.g., $r=0.24$ between TPJ and BA37) and connectivity network (e.g., $r=0.01$ between TPJ and BA37). This finding implies that these areas are activated similarly, but do not represent similar information nor do they communicate with each other. Another example, social-cognitive areas correlate moderately to somatosensory-motor areas (e.g., $r=0.32$ (representation), $r=0.59$ (activation) between MTG and BA1), except in the connectivity network (e.g., $r=0.03$ between MTG and BA1). As the last example, visual area V5 shows a moderate correlation to other brain areas in the representation network (e.g., $r=0.39$ between V5 and BA19) while a much stronger correlation is found in the other networks (e.g., $r=0.76$ (activation) $r=0.61$ (connectivity) between V5 and BA19).

For visualization purposes, we performed MDS on the three types of dissimilarity matrices to reconstruct two-dimensional spatial configuration that reflects the proximity in the matrices. Moreover, Procrustes transformations were performed to align the configurations. The resulting configurations are shown in Figure 6. The results confirm the high similarity ( $d$ (Procrustes distance: the difference between the shape of the two networks) between the activation and representation network $=0.48, d$ between the connectivity and representation network $=0.34, d$ between the activation and connectivity network $=0.42$ ) and some dissimilarities between the networks as was previously 


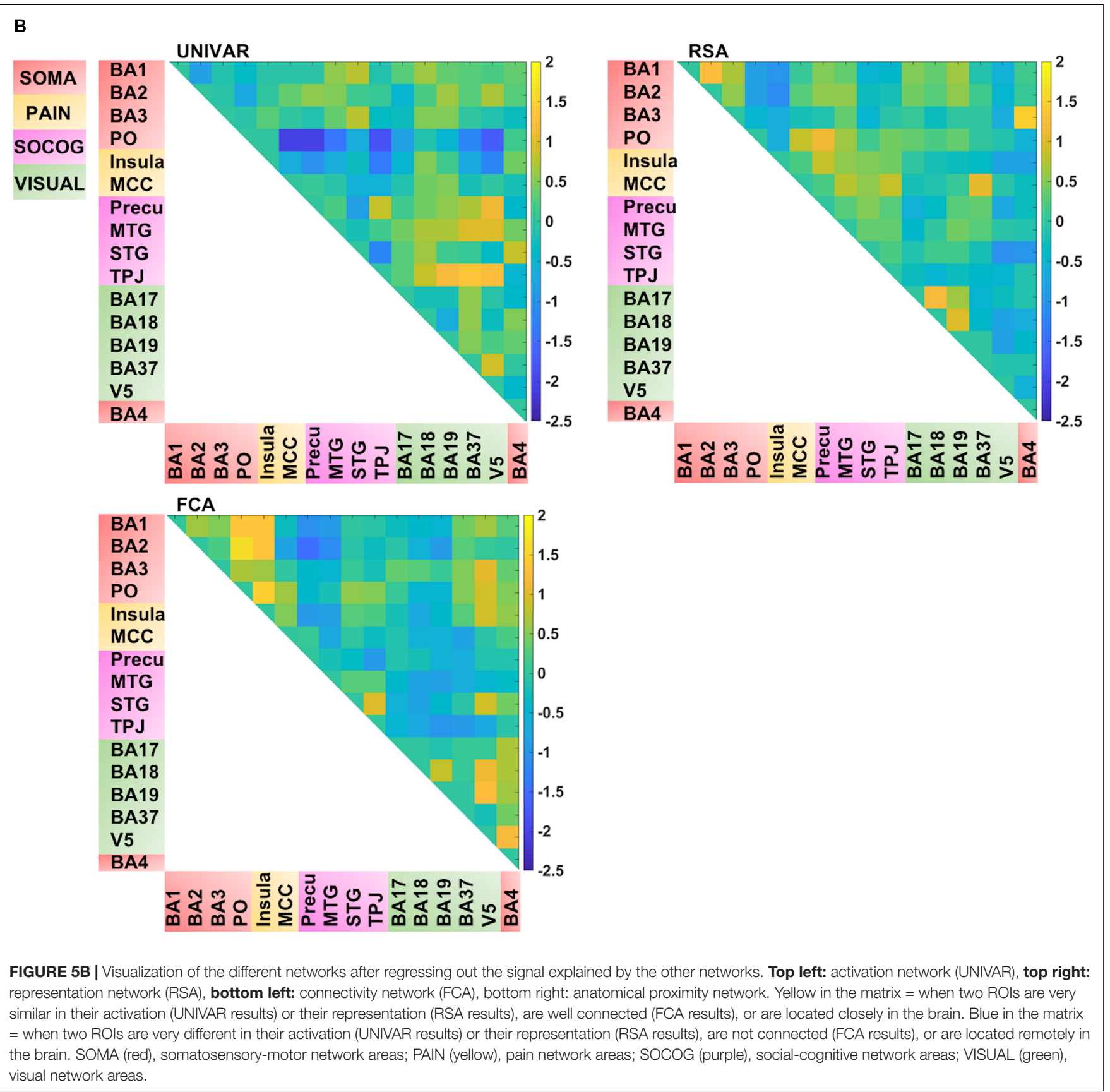

indicated by the (partial) correlation and multiple regression models. As an example of correspondence between the three networks, Figure 6 shows that somatosensory-motor areas are located nearby in all three networks, implying high similarity in activation and representation and strong interregional communication among these areas. As an example of a difference between the networks, the social-cognitive brain areas are placed close to visual areas overall in the activation network (blue in Figure 6) but not so much in the other networks. It suggests that social cognitive brain areas and the visual cortex do not represent the same information and that those areas are not functionally connected despite the similar magnitude of neural response. Other visualization methods such as principal component analysis result in similar plots as Figure 6.

\section{Summary}

The representation, the activation, and connectivity networks were proven to be reliable based on their signal-to-noise ratio. All three functional networks are organized highly similarly. For example, moderate correlations between social-cognitive areas and visual areas can be found in the representation, activation and connectivity network. Based on multiple regression models, we found that for each type of network, a lot of the organization 


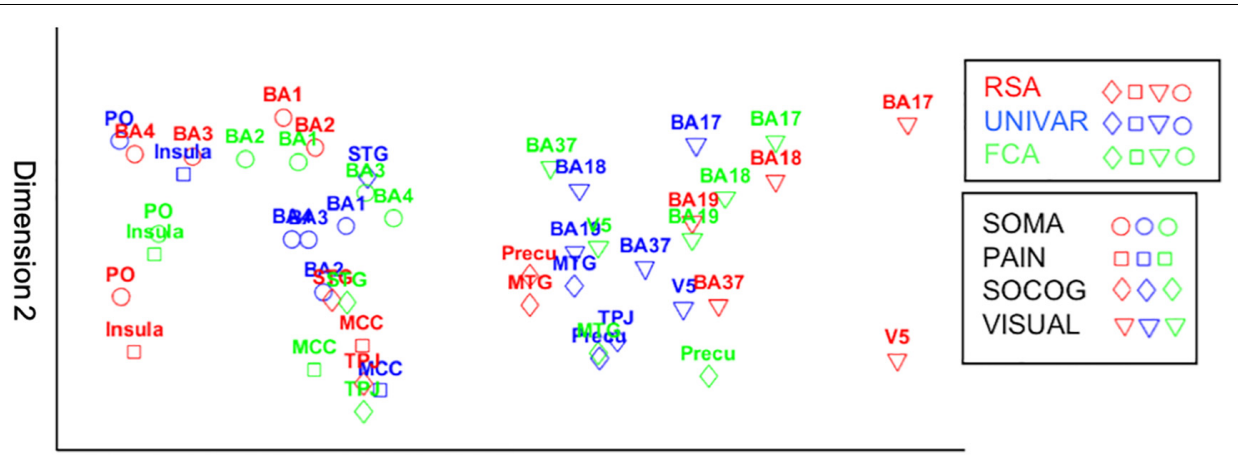

Dimension 1

FIGURE 6 | Procrustes transformed MDS results of the activation (UNIVAR, blue) and connectivity network (FCA, green) to the MDS results of the representation network (RSA, red). SOMA (circles), somatosensory-motor network areas; PAIN (squares), pain network areas; SOCOG (diamonds), social-cognitive network areas; VISUAL (triangles), visual network areas.

can be predicted from the other networks, but there is also some remaining variance left unexplained. For example, socialcognitive areas and somatosensory-motor areas are activated similarly (UNIVAR) and represent similar information (RSA), but do not communicate with each other (FCA). In other words, the networks derived from RSA, UNIVAR, and FCA, although highly similar, also each contain unique information. We have visualized these results using Procrustes transformations on MDS results in Figure 6.

\section{DISCUSSION}

RSA has recently emerged as a method for investigating how brain regions are organized into networks. UNIVAR and FCA are two other popular methods for analyzing fMRI data to understand functional networks. Although two or more of these methods have been used simultaneously to analyze the same set of data in many studies, most of them have focused on the properties of each ROI separately. No study, to our knowledge, directly and simultaneously compared networks derived from RSA with networks built with UNIVAR and FCA. In the current study, we explored how the organization of networks built from RSA, UNIVAR, and FCA relate to each other after ruling out the effect of the anatomical location of network nodes (ROIs). We analyzed fMRI data of a previous study (Lee Masson et al., 2018) with these methods and performed (partial) correlation and multiple regression analysis on the resulting networks.

The current study reveals that neural networks resulting from RSA, UNIVAR, and FCA are highly similar even after ruling out the effect of anatomical proximity. As predicted, brain areas within the somatosensory-motor network are similarly activated, represent similar task-related information, and are intrinsically connected. This also applies to the other sub-networks (pain, social-cognitive and visual). As outlined in the introduction, RSA, UNIVAR, and FCA share theoretical and/or methodological properties that can explain similarities as observed in this study. The high similarity in the neural networks of RSA and FCA provides support for the idea that brain areas showing similar stimulus-related selectivity are also intrinsically connected. Our finding is in line with previous resting-state fMRI studies that have identified functionally relevant networks, such as the primary visual network, auditory network, motor network, and cognitive networks, during rest (e.g., Biswal et al., 1995; Fox and Raichle, 2007; Jung et al., 2018).

On the other hand, our finding suggests that the network derived from each method contains unique signals. To reveal this, we compared the explainable variance of each network revealed by SNR estimation with the actual variance explained by the other networks. These results suggested that the network, derived from each method, contains unique information that none of the other networks are able to explain. Analyzing the remaining signal variance that was left unexplained, we were also able to reveal unique network organizations of each method. For example, brain areas in the social-cognitive network are similar to areas in the visual network in terms of neural activation, whereas neural patterns of those two sub-networks do not represent the same information and they are not intrinsically connected. Another example is the moderate correlations between social-cognitive brain areas and somatosensory-motor areas in the activation and representation network, but not in the connectivity network.

This unique information is important to keep in mind when interpreting a functional network found with one particular method. Although second-order RSA can be used to construct brain connectivity, RSA and FCA adopt different approaches shown in their methodology: correlating RDMs in RSA; correlating the BOLD signal fluctuations in FCA. Thus, RSA is used for investigating the similarity between brain areas in how they represent task-related information while FCA is used for investigating how a series of brain areas construct the intrinsically connected cortical network. These distinctions allow RSA and FCA to tap into the functional architecture of the brain from different perspectives as revealed in the unique information the networks contain.

Likewise, the same reasoning can be applied to the relationship between UNIVAR and FCA, and RSA and UNIVAR. As outlined in the introduction, they are related theoretically and empirically 
while they differ in their focus, allowing both similarities and dissimilarities between the resulting networks.

Such distinctions between networks derived from different methods have also been observed in the recent study of Jung and her colleagues (Jung et al., 2018) comparing resting-state fMRI and structural connectivity. Although their comparison involves different methods than ours, they provided some possible explanations that should be considered in the current study. The quality and nature of the datasets used for three methods (even from identical data sources, but measured at different times or analyzed in a different way) may not be equal and different measurement noise may be present (Jung et al., 2018). In addition, they mention that networks during mental activity are modulated away (slightly) from intrinsic connections, which is especially relevant to the comparison of RSA with FCA. Accordingly, our findings of similarities and differences between RSA and FCA networks are consistent with the observation that studies using both RSA and FCA lead to either similar or different conclusions about brain function derived from the two methods (e.g., Boets et al., 2013; Zeharia et al., 2015). Several other factors could play a role in the (dis)similarities between the networks derived from RSA, FCA, and UNIVAR, for example vasculature. Despite the high similarity across the networks derived from the UNIVAR, RSA, and FCA methods, given the nature of uniqueness of each network, we encourage researchers to understand the benefits of each methodology and what they (do not) detect; and to use them adequately depending on the research questions.

As a critical note, we point to several limitations of our current study. First, the current findings are based on only one task domain (i.e., social touch scene perception), and our conclusions should be complemented by future studies that include other tasks, such as moral decision-making tasks, or tasks using other sensory modalities such as auditory and tactile scenes. RSA and UNIVAR methods may not produce similar network organizations in another task. This could in particular be the case, when having a task with no activation differences across the conditions but evoking neural pattern selectivity. We hypothesize that it will be a general phenomenon; that also in other domains there will be a shared network organization that dominates with, in addition, a smaller unique component. Second, we selected a limited number of ROIs rather than including a large number of network nodes. One important argument for doing this is that the selected brain regions had to include meaningful task-related signals for performing RSA (see the description of diagonal vs. non-diagonal measures as a reliability test in choosing ROIs in Methods). The effect of the number and size of ROIs on the relationships between the networks obtained using RSA, UNIVAR, and FCA can be explored further. Finally, extending the comparisons made in the current study is another important step to take. Specifically, networks built from second-order RSA and multivariate functional connectivity could also be compared (Coutanche and Thompson-Schill, 2014; Anzellotti and Coutanche, 2018). Other analyses than RSA exist that can pick up on more complex spatial relations between ROIs (e.g., Haxby et al., 2011; Ozay et al., 2012; Firat et al., 2013; Onal et al., 2017). Although RSA is one of the most commonly used approaches in cognitive neuroscience in comparing neural spaces across different brain regions, uncovering the complexity of the spatial relationships captured through more advanced approaches may help to compare the three methodologies in depth.

\section{CONCLUSION}

The present study provides first-time evidence that cortical networks derived from three commonly used neuroimaging approaches (RSA, UNIVAR, and FCA) are highly similar regardless of the structural variations of each network. Importantly, the study also demonstrates that each of these three networks contains unique information, unexplainable by the other networks. As such, all three methods are important when investigating the functional organization of networks in the brain. Improving the understanding of the relationship between the functional networks derived from these methods will allow researchers to use RSA, UNIVAR, and FCA more adequately.

\section{DATA AVAILABILITY STATEMENT}

The data for this study can be found in the Open Science Framework (https://osf.io/b4np9/).

\section{ETHICS STATEMENT}

All participants provided written informed consent before the experiment in accordance with the Declaration of Helsinki. The study was carried out in accordance with the recommendations of and approved by the Medical Ethical Committee of KU Leuven (S53768 and S59577).

\section{AUTHOR CONTRIBUTIONS}

IP, HO, and HL set up the project, fMRI data collection (see Lee Masson et al., 2018), and wrote the manuscript. IP and HL analyzed the data.

\section{FUNDING}

This work was supported by the European Research Council (Grant No. ERC-2011-Stg-284101), a federal research action (Grant No. IUAP-P7/11), Fonds Wetenschappelijk Onderzoek Vlaanderen (Grant No. G088216N), a Hercules Grant (Grant No. ZW11_10), and an Excellence of Science grant (Grant No. G0E8718N) to HO, and Marguerite-Marie Delacroix Foundation (GV/B-336) to HL.

\section{ACKNOWLEDGMENTS}

This manuscript has been released as a preprint at BioRxiv (doi: 10.1101/487199). 


\section{REFERENCES}

Alakörkkö, T., Saarimäki, H., Glerean, E., Saramäki, J., and Korhonen, O. (2017). Effects of spatial smoothing on functional brain networks. Eur. J. Neurosci. 46, 2471-2480. doi: 10.1111/ejn.13717

Anzellotti, S., and Coutanche, M. N. (2018). Beyond functional connectivity: investigating networks of multivariate representations. Trends Cogn. Sci. 22, 258-269. doi: 10.1016/j.tics.2017.12.002

Bassett, D. S., Wymbs, N. F., Porter, M. A., Mucha, P. J., Carlson, J. M., and Grafton, S. T. (2011). Dynamic reconfiguration of human brain networks during learning. PNAS 108, 7641-7646. doi: 10.1073/pnas.101898 5108

Biswal, B., Yetkin, F. Z., Haughton, V. M., and Hyde, J. S. (1995). Functional connectivity in the motor cortex of resting human brain using echo-planar MRI. Magn. Reson. Med. 34, 537-541. doi: 10.1002/mrm.1910340409

Boets, B., Op De Beeck, H. P., Vandermosten, M., Scott, S. K., Gillebert, C. R., Mantini, D., et al. (2013). Intact but less accessible phonetic representations in adults with dyslexia. Science 342, 1251-1254. doi: 10.1126/science.1244333

Bulthé, J., Prinsen, J., Vanderauwera, J., Duyck, S., Daniels, N., Gillebert, C. R., et al. (2018). Multi-method brain imaging reveals impaired representations of number as well as altered connectivity in adults with dyscalculia. Neuroimage 190, 289-302. doi: 10.1016/j.neuroimage.2018.06.012

Cordes, D., Haughton, V. M., Arfanakis, K., Carew, J. D., Turski, P. A., Moritz, C. H., et al. (2001). Frequencies contributing to functional connectivity in the cerebral cortex in "resting-state" data. AJNR Am. J. Neuroradiol. 22, 1326-1333.

Cordes, D., Haughton, V. M., Arfanakis, K., Wendt, G. J., Turski, P. A., Moritz, C. H., et al. (2000). Mapping functionally related regions of brain with functional connectivity MR imaging. AJNR Am. J. Neuroradiol. 21, 1636-1644.

Coutanche, M. N. (2013). Distinguishing multi-voxel patterns and mean activation: why, how, and what does it tell us? Cogn. Affect. Behav. Neurosci. 13, 667-673. doi: 10.3758/s13415-013-0186-2

Coutanche, M. N., and Thompson-Schill, S. L. (2014). Using informational connectivity to measure the synchronous emergence of fMRI multi-voxel information across time. J. Vis. Exp. 89:e51226. doi: 10.3791/51226

Crossley, N. A., Mechelli, A., Vértes, P. E., Winton-Brown, T. T., Patel, A. X., Ginestet, C. E., et al. (2013). Cognitive relevance of the community structure of the human brain functional coactivation network. PNAS 110, 11583-11588. doi: $10.1073 /$ pnas. 1220826110

Davis, T., LaRocque, K. F., Mumford, J. A., Norman, K. A., Wagner, A. D., and Poldrack, R. A. (2014). What do differences between multi-voxel and univariate analysis mean? How subject-, voxel-, and trial-level variance impact fMRI analysis. Neuroimage 97, 271-283. doi: 10.1016/j.neuroimage.2014. 04.037

Ebisch, S. J. H., Mantini, D., Romanelli, R., Tommasi, M., Perrucci, M. G., Romani, G. L., et al. (2013). Long-range functional interactions of anterior insula and medial frontal cortex are differently modulated by visuospatial and inductive reasoning tasks. Neuroimage 78, 426-438. doi: 10.1016/j.neuroimage.2013.04. 058

Eickhoff, S. B., Stephan, K. E., Mohlberg, H., Grefkes, C., Fink, G. R., Amunts, K., et al. (2005). A new SPM toolbox for combining probabilistic cytoarchitectonic maps and functional imaging data. Neuroimage 25, 1325-1335. doi: 10.1016/j. neuroimage.2004.12.034

Fair, D. A., Schlaggar, B. L., Cohen, A. L., Miezin, F. M., Dosenbach, N. U. F., Wenger, K. K., et al. (2007). A method for using blocked and event-related fMRI data to study "resting state" functional connectivity. Neuroimage 35, 396-405. doi: 10.1016/j.neuroimage.2006.11.051

Firat, O., Ozay, M., Onal, I., Öztekin, I., and Yarman-Vural, F. T. (2013). Functional mesh learning for pattern analysis of cognitive processes. ICCI-CC 2013, 161167. doi: 10.1109/ICCI-CC.2013.6622239

Fox, M. D., Corbetta, M., Snyder, A. Z., Vincent, J. L., and Raichle, M. E. (2006). Spontaneous neuronal activity distinguishes human dorsal and ventral attention systems. PNAS 103, 10046-10051. doi: 10.1073/pnas.0604187103

Fox, M. D., and Raichle, M. E. (2007). Spontaneous fluctuations in brain activity observed with functional magnetic resonance imaging. Nat. Rev. Neurosci. 8, 700-711. doi: 10.1038/nrn2201

Friston, K. J. (1994). Functional and effective connectivity in neuroimaging: a synthesis. Hum. Brain Mapp. 2, 56-78. doi: 10.1002/hbm.460020107
Friston, K. J. (2011). Functional and effective connectivity: a review. Brain Connect. 1, 13-36. doi: 10.1089/brain.2011.0008

Geerligs, L., Henson, R. N., and Cam-Can. (2016). Functional connectivity and structural covariance between regions of interest can be measured more accurately using multivariate distance correlation. Neuroimage 135, 16-31. doi: 10.1016/j.neuroimage.2016.04.047

Gilron, R., Rosenblatt, J., Koyejo, O., Poldrack, R. A., and Mukamel, R. (2017). What's in a pattern? Examining the type of signal multivariate analysis uncovers at the group level. Neuroimage 146, 113-120. doi: 10.1016/j.neuroimage.2016. 11.019

Gordon, I., Voos, A. C., Bennett, R. H., Bolling, D. Z., Pelphrey, K. A., and Kaiser, M. D. (2013). Brain mechanisms for processing affective touch. Hum. Brain Mapp. 34, 914-922. doi: 10.1002/hbm.21480

Haxby, J. V. (2012). Multivariate pattern analysis of fMRI: the early beginnings. Neuroimage 62, 852-855. doi: 10.1016/j.neuroimage.2012.03.016

Haxby, J. V., Gobbini, M. I., Furey, M. L., Ishai, A., Schouten, J. L., and Pietrini, P. (2001). Distributed and overlapping representations of faces and objects in ventral temporal cortex. Science 293, 2425-2430. doi: 10.1126/science.106 3736

Haxby, J. V., Guntupalli, J. S., Connolly, A. C., Halchenko, Y. O., Conroy, B. R., Gobbini, M. I., et al. (2011). A common, high-dimensional model of the representational space in human ventral temporal cortex. Neuron 72, 404-416. doi: 10.1016/j.neuron.2011.08.026

Haynes, J. D. (2015). A primer on pattern-based approaches to fMRI: principles, pitfalls, and perspectives. Neuron 87, 257-270. doi: 10.1016/j.neuron.2015.05. 025

Haynes, J. D., and Rees, G. (2006). Decoding mental states from brain activity in humans. Nat. Rev. Neurosci. 7, 523-534. doi: 10.1038/nrn1931

Hendriks, M. H. A., Daniels, N., Pegado, F., and Op de Beeck, H. P. (2017). The effect of spatial smoothing on representational similarity in a simple motor paradigm. Front. Neurol. 8:222. doi: 10.3389/fneur.2017.00222

Jacoby, N., Bruneau, E., Koster-Hale, J., and Saxe, R. (2016). Localizing pain matrix and theory of mind networks with both verbal and non-verbal stimuli. Neuroimage 126, 39-48. doi: 10.1016/j.neuroimage.2015.11.025

Jimura, K., and Poldrack, R. A. (2012). Analyses of regional-average activation and multivoxel pattern information tell complementary stories. Neuropsychologia 50, 544-552. doi: 10.1016/j.neuropsychologia.2011.11.007

Jung, J., Visser, M., Binney, R. J., and Lambon Ralph, M. A. (2018). Establishing the cognitive signature of human brain networks derived from structural and functional connectivity. Brain Struct. Funct. 223, 4023-4038. doi: 10.1007/ s00429-018-1734- $\mathrm{x}$

Kaas, J. H. (1997). Topographic maps are fundamental to sensory processing. Brain Res. Bull. 44, 107-112. doi: 10.1016/S0361-9230(97)00094-4

Kriegeskorte, N. (2011). Pattern-information analysis: from stimulus decoding to computational-model testing. Neuroimage 56, 411-421. doi: 10.1016/j. neuroimage.2011.01.061

Kriegeskorte, N., Mur, M., and Bandettini, P. (2008). Representational similarity analysis - connecting the branches of systems neuroscience. Front. Syst. Neurosci. 2:4. doi: 10.3389/neuro.06.004.2008

Lamm, C., and Majdandžić, J. (2015). The role of shared neural activations, mirror neurons, and morality in empathy - A critical comment. Neurosci. Res. 90, 15-24. doi: 10.1016/j.neures.2014.10.008

Lee Masson, H., and Op de Beeck, H. (2018). Socio-affective touch expression database. PLoS One 13:e0190921. doi: 10.1371/journal.pone.0190921

Lee Masson, H., Van De Plas, S., Daniels, N., and Op de Beeck, H. (2018). The multidimensional representational space of observed socio-affective touch experiences. Neuroimage 175, 297-314. doi: 10.1016/j.neuroimage.2018.04.007

Logothetis, N. K. (2008). What we can do and what we cannot do with fMRI. Nature 453, 869-878. doi: 10.1038/nature06976

Maldjian, J. A., Laurienti, P. J., Kraft, R. A., and Burdette, J. H. (2003). An automated method for neuroanatomic and cytoarchitectonic atlas-based interrogation of fMRI data sets. Neuroimage 19, 1233-1239. doi: 10.1016/ S1053-8119(03)00169-1

Mars, R. B., Sallet, J., Schüffelgen, U., Jbabdi, S., Toni, I., and Rushworth, M. F. S. (2012). Connectivity-based subdivisions of the human right "temporoparietal junction area": evidence for different areas participating in different cortical networks. Cereb. Cortex 22, 1894-1903. doi: 10.1093/cercor/ bhr268 
Morrison, I., Björnsdotter, M., and Olausson, H. (2011). Vicarious responses to social touch in posterior insular cortex are tuned to pleasant caressing speeds. J. Neurosci. 31, 9554-9562. doi: 10.1523/JNEUROSCI.0397-11. 2011

Mur, M., Bandettini, P. A., and Kriegeskorte, N. (2009). Revealing representational content with pattern-information fMRI - An introductory guide. Soc. Cogn. Affect. Neurosci. 4, 101-109. doi: 10.1093/scan/nsn044

Norman, K. A., Polyn, S. M., Detre, G. J., and Haxby, J. V. (2006). Beyond mindreading: multi- voxel pattern analysis of fMRI data. Trends Cogn. Sci. 10, 424-430. doi: 10.1016/j.tics.2006.07.005

Onal, I., Ozay, M., Mizrak, E., Öztekin, I., and Yarman-Vural, F. T. (2017). A new representation of $\mathrm{AMRI}$ signal by a set of local meshes for brain decoding. IEEE Trans. Signal. Inform. Process. Over Netw. 3, 683-694. doi: 10.1109/TSIPN.2017. 2679491

Ozay, M., Öztekin, I., Öztekin, U., and Yarman-Vural, F. T. (2012). Mesh learning for classifying cognitive processes. arXiv. [preprint]. doi: 10.1109/EMBC.2013. 6611113

Raizada, R. D. S., and Kriegeskorte, N. (2010). Pattern-information fMRI: new questions which it opens up and challenges which face it. Int. J. Imaging Syst. Technol. 20, 31-41. doi: 10.1002/ima.20225

Raizada, R. D. S., Tsao, F. M., Liu, H. M., and Kuhl, P. K. (2010). Quantifying the adequacy of neural representations for a cross-language phonetic discrimination task: prediction of individual differences. Cereb. Cortex 20, 1-12. doi: 10.1093/cercor/bhp076

Ritchie, J. B., Bracci, S., and Op de Beeck, H. (2017). Avoiding illusory effects in representational similarity analysis: what (not) to do with the diagonal. Neuroimage 14, 197-200. doi: 10.1016/j.neuroimage.2016.1 2.079

Rolls, E. T., O’Doherty, J., Kringelbach, M. L., Francis, S., Bowtell, R., and McGlone, F. (2003). Representations of pleasant and painful touch in the human orbitofrontal and cingulate cortices. Cereb. Cortex 13, 308-317. doi: 10.1093/cercor/13.3.308

Salvador, R., Suckling, J., Coleman, M. R., Pickard, J. D., Menon, D., and Bullmore, E. (2005). Neurophysiological architecture of functional magnetic resonance images of human brain. Cereb. Cortex 15, 1332-1342. doi: 10.1093/cercor/ bhi016

Whitfield-Gabrieli, S., and Nieto-Castanon, A. (2012). CONN: a functional connectivity toolbox for correlated and anticorrelated brain networks. Brain Connect. 2, 125-141. doi: 10.1089/brain.2012.0073

Xue, S. W., Weng, X. C., He, S., and Li, D. W. (2013). Similarity representation of pattern-information fMRI. Chin. Sci. Bull. 58, 1236-1242. doi: 10.1007/s11434013-5743-0

Yang, Z., Fang, F., and Weng, X. (2012). Recent developments in multivariate pattern analysis for functional MRI. Neurosci. Bull. 28, 399-408. doi: 10.1007/ s12264-012-1253-3

Zeharia, N., Hertz, U., Flash, T., and Amedi, A. (2015). New whole-body sensory-motor gradients revealed using phase-locked analysis and verified using multivoxel pattern analysis and functional connectivity. J. Neurosci. 35, 2845-2859. doi: 10.1523/JNEUROSCI.4246-14.20

Conflict of Interest: The authors declare that the research was conducted in the absence of any commercial or financial relationships that could be construed as a potential conflict of interest.

Copyright (C) 2020 Pillet, Op de Beeck and Lee Masson. This is an open-access article distributed under the terms of the Creative Commons Attribution License (CC BY). The use, distribution or reproduction in other forums is permitted, provided the original author(s) and the copyright owner(s) are credited and that the original publication in this journal is cited, in accordance with accepted academic practice. No use, distribution or reproduction is permitted which does not comply with these terms. 